\title{
Desempenho de genótipos de melancia de frutos pequenos em diversas densidades de plantio
}

\author{
Anamaria RP Ramos'; Rita de Cássia S Dias²; Carlos Alberto Aragão; Patrício F Batista1; Mayara \\ Milena da L Pires ${ }^{1}$ \\ 'UNEB-DTCS, Av. Edgard Chastinet, s/n, 48900-000 Juazeiro-BA; anamaria-ramos@oi.com.br; carlosaragao@hotmail.com; milamlp@ \\ gmail.com; ${ }^{2}$ Embrapa Semiárido, C. Postal 23, 56300-000 Petrolina-PE; ritadias@cpatsa.embrapa.br
}

\section{RESUMO}

O experimento foi realizado em campo da UNEB, Juazeiro (BA). Foram avaliados três espaçamentos combinados com dez genótipos de melancia diplóides, sendo oito híbridos experimentais, provenientes do Programa de Melhoramento Vegetal da Embrapa Semiárido e duas cultivares comerciais (Sugar Baby e Smile), quanto ao desempenho agronômico e qualidade de frutos. Os híbridos experimentais Hib 02, Hib 03, Hib 04, Hib 05 e Hib 07 apresentaram as melhores taxas de emergência sendo que os híbridos 02, 03 e 06 estiveram entre os melhores para altura e diâmetro do colo das plantas aos 21 dias e para massa fresca da parte aérea no final do ciclo. Os espaçamentos utilizados não afetaram o desenvolvimento das plantas de melancia, como também não afetaram a qualidade fisiológica dos frutos dos genótipos avaliados. De maneira geral, não foram observadas diferenças com relação à qualidade dos frutos entre os híbridos e as cultivares, mostrando-se dessa forma bastante promissores para o seu futuro lançamento no mercado.

Palavras-chave: Citrullus lanatus, espaçamento, cultivares diplóides.

\begin{abstract}
Performance of watermelon genotypes of small fruit under several planting densities

The experiment was carried out on the experimental field of the Bahia State University in Juazeiro, Bahia state, Brazil. Three different spacings were assessed, combined with ten diploid watermelon cultivars, eight of which were experimental hybrids, originated from the Plant Breeding Program of Embrapa Semiárido, and two commercial cultivars (Sugar Baby and Smile) for agronomical performance and post-harvest fruit quality. The experimental hybrids Hyb 02, Hyb 03, Hyb 04, Hyb 05 and Hyb 07 showed the best emergency rates, with outstanding results for the hybrids 02,03 and 06 , which were among the best considering the seedling height and colon diameter besides fresh weight of shoot. The different spacings did not influence the plant development or the physiological quality of the fruit of the genotypes assessed.
\end{abstract}

Keywords: Citrullus lanatus, spacing, diploid cultivars.

(Recebido para publicação em 11 de fevereiro de 2011; aceito em 22 de maio de 2012) (Received on February 11, 2011; accepted on May 22, 2012)

\begin{abstract}
A melancia (Citrullus lanatus) é uma cucurbitácea originária provavelmente, da África tropical e possui propriedades nutricionais e terapêuticas, aumentando o interesse de vários segmentos da sociedade pelo seu fruto (Andrade Junior, 2004). É de grande expressão econômica e social. Atualmente, destacam-se como maiores produtores os estados do Rio Grande do Sul, São Paulo, Bahia, Rio Grande do Norte e Tocantins, que contribuem com mais de $60 \%$ da produção nacional (AGRIANUAL, 2007). O Nordeste brasileiro é responsável por $23 \%$ de toda a produção nacional (AGRIANUAL, 2007), porém nessa região o cultivo é uma atividade de risco, seja do ponto de vista dos preços ou da produtividade que se mostra também bastante variável, em função dos problemas agronômicos, destacando-se a carência de informações sobre o manejo adequado para a cultura
\end{abstract}

na região Nordeste.

No Brasil, a preferência do mercado consumidor leva em consideração o tamanho e formato do fruto, coloração da polpa, teor de sólidos solúveis e presença ou ausência de sementes. Mais recentemente, destaca-se o surgimento de novos tipos de melancias, as chamadas mini-melancias. Isto se deve principalmente à exigência do mercado por produtos alternativos, onde o consumidor opta por frutos menores, sem sementes e de excelente qualidade. Observa-se que, na maior parte das áreas plantadas, a predominância é de frutos grandes, com peso médio acima de 6,0 kg e segundo Dias et al. (2001), no Vale do Submédio do São Francisco, a cultivar Crimson Sweet, predomina nos cultivos.

O desenvolvimento vegetativo tem importância para os produtores, pois, associado à prolificidade, determina a estratégia que poderá ser adotada na densidade de plantio e repercute no tamanho dos frutos e na produtividade. No tocante à densidade de plantio, as pressões exercidas pela população de plantas afetam de modo marcante o seu desenvolvimento (Resende \& Costa, 2003).

Poucos trabalhos sobre correlações entre caracteres de melancia têm sido relatados (Ferreira et al., 2003). Desta forma, a seleção de bons genótipos para uma determinada região, deve se basear em avaliações como vigor das sementes, poder de germinação e no posterior crescimento, desenvolvimento e produção das plantas.

$\mathrm{Na}$ melancia, ao se aumentar o espaçamento entre plantas, ocorre uma diminuição na produtividade comercial, mas há um aumento no peso médio dos frutos (Halsey, 1959; Patil \& Bhosale, 1976; Brinen et al., 1979). Segundo 
Brinen \& Locascio (1979), a produtividade de frutos comerciais decresceu, ao passo que o peso médio de fruto aumentou com o incremento do espaçamento entre plantas de $0,6 \mathrm{~m}$ para 2,4 m. Srinivas et al. (1991) concluíram que a produtividade de frutos de melancia aumentou de 33,6 $\mathrm{t} \mathrm{ha}^{-1}$ para 38,9 $\mathrm{tha}^{-1}$ em 1984 e de 30,3 t ha ${ }^{-1}$ para 36,2 $\mathrm{t} \mathrm{ha}^{-1}$ em 1985, quando a população de plantas passou de 11.111 para 16.666 plantas $\mathrm{ha}^{-1}$, respectivamente. Segundo NeSmith (1993), a produtividade total e comercial de frutos de melancia aumentou quando o espaçamento entre plantas decresceu de 2,2 $\mathrm{m}$ para $0,9 \mathrm{~m}$.

O cultivo convencional da melancia no Brasil constitui-se do plantio com duas plantas por cova e espaçamentos de 4,0 m x 2,0 m; 3,0 m x 2,5 m e 3,0 m x 2,0 m, o que proporciona a obtenção máxima de 3.333 plantas ha ${ }^{-1}$. Portanto, o que se verifica nesse sistema é a obtenção de uma baixa densidade de plantas por área, quando comparado ao cultivo tutorado. Quando cultivada em ambiente protegido e com uso de tutoramento vertical, tem-se a possibilidade do cultivo em diferentes épocas do ano, melhor qualidade do produto colhido, alem de possibilitar maior adensamento das plantas. Segundo Garcia (1998), cultivando melancia no sistema tradicional (rasteiro), a diminuição no espaçamento entre plantas proporciona aumento na produtividade, mas, ocorre diminuição no tamanho dos frutos, o que às vezes é desejável.

Santos et al. (2002), avaliando a produção dos híbridos 'AF 1927', 'AF 1934', 'AF 1938' e 'AF 1939' de melancia tutorada sob as densidades de plantio de 10, 20, 30 e 40 mil plantas ha-1, em ambiente protegido, verificaram que na densidade de 35 mil plantas ha ${ }^{-1}$ obteve-se melhores resultados para os híbridos estudados, proporcionando menor peso de frutos e maior produtividade.

Avaliando diferentes espaçamentos de plantio na produção da cultivar Crimson Sweet no município de Petrolina (PE), Rezende \& Costa (2003) verificaram que o incremento dos espaçamentos, tanto entre linhas como entre plantas, produziu frutos de maior tamanho, tendo o espaçamento 3,0 x 0,8 $\mathrm{m}$ apresentado a maior massa fresca do fruto $\left(8,8 \mathrm{~kg}\right.$ fruto $\left.^{-1}\right)$ e o maior número de frutos por planta (1,35 frutos). A maior produtividade foi encontrada no espaçamento de 3,0 m entre linhas (42,5 $\left.\mathrm{t} \mathrm{ha}^{-1}\right)$. Nos espaçamentos de 0,6 e 0,8 $\mathrm{m}$ entre plantas obtiveram as maiores produtividades com 42,5 e 45,3 $\mathrm{t} \mathrm{ha}^{-1}$, respectivamente, não mostrando diferenças estatísticas entre si. O menor espaçamento entre plantas proporcionou maior produção de refugos (sendo considerado neste caso, os frutos com menos de $6 \mathrm{~kg}$ ), com 20,2 t ha-1, seguido pelos espaçamentos de $0,6 \mathrm{~m}\left(12,9 \mathrm{t} \mathrm{ha}^{-1}\right) \mathrm{e}$ 0,8 m (8,6 tha-1).

Segundo Singh \& Naik (1989), características de qualidade de frutos na cultura da melancia como sólidos solúveis (SS) não foram influenciadas significativamente pelos espaçamentos ( $2,0 \times 0,8 \mathrm{~m} ; 2,0 \times 1,0 \mathrm{~m}$ e $2,0 \times 1,2 \mathrm{~m})$ e doses de nitrogênio $(50,100,150$ e 200 $\mathrm{kg} \mathrm{ha}^{-1}$ ), testados em dois ciclos da cultura da melancia. Hedge (1988) também não observou efeito significativo sobre a porcentagem de SS quando aplicou 60 , 120 e $180 \mathrm{~kg} \mathrm{ha}^{-1}$ de N.

Santos Filha et al. (2005) analisaram o comportamento de dois genótipos de melancia sem sementes (W 913 e SQ) no município de Mossoró e constataram diferenças significativas entre os mesmos para as características $\mathrm{pH}$, sólidos solúveis, firmeza e acidez titulável, sendo que estas diferenças podem ser atribuídas a aspectos intrínsecos de cada genótipo.

O objetivo do trabalho foi avaliar o desempenho agronômico e a qualidade pós-colheita de cultivares e híbridos experimentais de melancia nas condições do Vale do São Francisco, submetidos a diferentes espaçamentos de plantio.

\section{MATERIAL E MÉTODOS}

O experimento foi realizado no segundo semestre de 2006, em campo experimental da Universidade do Estado da Bahia (UNEB), situado no município de Juazeiro, Bahia.

Foram avaliados três espaçamentos (0,3 x 2,0 m; 0,4 x 2,0 m; 0,5 x 2,0 m), combinados com dez genótipos de melancia, sendo oito híbridos diplóides experimentais, provenientes do Programa de Melhoramento Vegetal da Embrapa Semiárido onde foram obtidas linhas de melancia com resistência ao oídio ("LDROCPATSA") e linhas a partir das cultivares comerciais Sugar Baby e Smile ("LDSB" e "LDSM"), que foram comparados com as cultivares comerciais Hib 01 (LDRO5CPATSA x LD3SB); Hib 02 (LDRO5CPATSA x LD2SM); Hib 03 (LDRO4CPATSA x LD1SB); Hib 04 (LDRO4CPATSA x LD2SB); Hib 05 (LDRO3 CPATSA x LD3SB); Hib 06 (LD1SM X LDRO2CPATSA); Hib 07 (LD1SB x LDRO1CPATSA); Hib 08 (LD1SM x LDRO3 CPATSA); Smile e Sugar Baby.

O delineamento experimental utilizado foi blocos ao acaso, em parcelas subdivididas com 3 repetições, considerando-se os genótipos como parcelas e os espaçamentos como subparcelas. Os tratamentos resultaram da combinação de três espaçamentos e dez genótipos de melancia (oito experimentais e duas cultivares comerciais usadas como testemunhas), com quatro plantas por subparcela.

O solo da área do ensaio é classificado como Neossolo flúvico com as características químicas e físicas na camada de 0-20 cm: $\mathrm{K}=0,25 ; \mathrm{Ca}=1,5$; $\mathrm{Mg}=0,5$ e $\mathrm{Na}=0,01 \mathrm{cmol}_{\mathrm{c}} \mathrm{dm}^{-3} ; \mathrm{P}=41$ $\mathrm{mg} \mathrm{dm}{ }^{-3}$; areia $=536 \mathrm{~g} \mathrm{~kg}^{-1}$; silte $=365$ $\mathrm{g} \mathrm{kg}^{-1}$; argila $=99 \mathrm{~g} \mathrm{~kg}^{-1}$ e $\mathrm{pH}$ em $\mathrm{H}_{2} 0$ $=6,7$.

Após a aração e gradagem fez-se a distribuição de esterco de caprino curtido na quantidade de $2,1 \mathrm{~kg}$ por linha de plantio, 50 dias antes do transplantio. Após este período, procedeu-se a abertura dos sulcos com aproximadamente $30 \mathrm{~cm}$ de profundidade e a adubação de plantio foi feita com base na análise do solo e na recomendação do Manual de Adubação do Estado da Bahia (1989), tendo aplicado $120 \mathrm{~kg} \mathrm{ha}^{-1}$ de nitrogênio (ureia); $80 \mathrm{~kg} \mathrm{ha}^{-1}$ de potássio (cloreto de potássio) e $80 \mathrm{~kg} \mathrm{ha}^{-1}$ de fósforo (superfosfato simples) em cobertura.

O plantio das sementes foi realizado em casa de vegetação, em bandejas de poliestireno expandido de 200 células, utilizando-se substrato comercial Plantmax $^{\circledR}$, a uma profundidade de 2 $\mathrm{cm}$ e, irrigadas diariamente, de maneira a manter o substrato sempre úmido até as mudas serem transplantadas para o 
campo. As plantas permaneceram em casa de vegetação coberta com sombrite de $50 \%$ por um período de 21 dias até o transplantio, quando estavam com duas folhas definitivas.

Foram feitas avaliações de emergência (\%), consistindo do cálculo de plantas normais (emergência) quantificadas de acordo com as regras para análises de sementes (RAS) (Brasil, 1992). Ao final de 21 dias contou-se o número de plantas normais emergidas a fim de obter a porcentagem de emergência de acordo com as RAS (Brasil, 1992).

A floração feminina foi avaliada 42 dias após o plantio, quando foi feita a primeira avaliação do comprimento do ramo principal, que foi repetido 7 e 14 dias depois da primeira avaliação, com o auxílio de uma trena.

As avaliações de crescimento foram realizadas aos 15 e 21 dias após a semeadura, sendo medidas, com o auxílio de régua milimetrada e paquímetro, a altura da planta (ALT) e diâmetro do colo (DIA).

As colheitas dos frutos foram realizadas em duas etapas: "cortes" aos 57 DAT (dias após o transplantio) e 64 DAT, utilizando-se como critérios de colheita o ciclo da cultura, mudança de coloração da região do fruto que fica em contato com o solo (de branca para amarelo claro) e do som "oco" emitido quando o fruto é tocado com os dedos. Os frutos foram colhidos, colocados em contentores e transportados para o laboratório do Setor de Olericultura do Departamento de Tecnologia e Ciências Sociais.

As variáveis determinadas nas plantas e nos frutos foram comprimento do ramo principal; massa fresca da parte aérea (g); massa média de frutos (divisão da produção total da unidade experimental pelo número de frutos da mesma, em gramas); produção por planta (massa total de frutos da área útil da unidade experimental pelo número de plantas úteis, em $\mathrm{kg}$ ), número de frutos por planta (divisão do número total de frutos pelo número de plantas da área útil da unidade experimental) e a produtividade total (determinada a partir da produção total de frutos da área útil da unidade experimental, em t/ha).
Foi utilizada uma balança digital para determinação da massa fresca da parte aérea, produtividade comercial e massa média de frutos (tabela 2).

Os frutos também foram avaliados quanto ao teor de sólidos solúveis (SS), acidez titulável (AT), relação entre $\mathrm{SS}$ / AT, firmeza da polpa e pH. O conteúdo de SS foi determinado por refratometria, utilizando-se o suco da polpa filtrado, homogeneizado em liquidificador doméstico e fazendo-se a leitura do filtrado em refratômetro portátil, modelo 103 , com leitura na faixa de 0 a $32^{\circ} \mathrm{Brix}$.

A acidez titulável (AT) foi determinada em duplicata, utilizando-se alíquota de $2 \mathrm{~g}$ de suco, ao qual se adicionaram $23 \mathrm{~mL}$ de água destilada e 3 gotas de fenolftaleína alcoólica a 1\%. A seguir, titulou-se até o ponto de viragem com solução de $\mathrm{NaOH}$ a $0,1 \mathrm{~N}$, previamente padronizada, sendo os resultados expressos em \% de ácido cítrico.

A medida da firmeza de polpa foi feita, utilizando-se um penetrômetro manual, tipo CAT 719-20, com ponteira de diâmetro de $8 \mathrm{~mm}$ de diâmetro. $\mathrm{O}$ fruto foi dividido longitudinalmente em duas partes, sendo realizadas três leituras em cada uma. As leituras foram feitas eqüidistantes e na região equatorial da polpa e os resultados expressos em Newton $(\mathrm{N})$.

O potencial hidrogeniônico $(\mathrm{pH})$ foi determinado pela imersão do eletrodo de pHmetro digital na solução obtida pela extração da polpa do fruto homogeneizada com uma centrífuga doméstica.

As análises de variâncias com teste $\mathrm{F}$, bem como as comparações pelo teste de Tukey, ao nível de 5\% de probabilidade, foram realizadas com auxílio do software SAS.

\section{RESULTADOS E DISCUSSÃO}

As maiores percentagens de emergência de plantas foram apresentadas nos genótipos 02, 05 e na cultivar Sugar Baby, com 100, 90 e 98\% respectivamente, sendo que os menores valores foram apresentados pelo híbrido 06 e pela cv. Smile com 55 e $50 \%$ respectivamente. As avaliações de altura de plantas e diâmetro de colo, realizadas aos 15 e 21 dias permitiram discriminar os genótipos testados (Tabela 1). Aos 21 dias, o genótipo 02 destacou-se em magnitude dos demais apresentando médias de alturas de plantas de $6,1 \mathrm{~cm}$ e diâmetro de colo de $2,3 \mathrm{~mm}$. Não diferiu dos genótipos 03, 06 e da cv. Smile para altura de plantas. Para o diâmetro do colo o genótipo 02 diferiu apenas dos genótipos 01 e das cultivares Smile e Sugar Baby. O desempenho baseado na emergência e crescimento de plantas (altura e diâmetro do colo) é um indicativo do potencial dos híbridos na geração de sementes de qualidade. A aceitação de um híbrido no mercado leva em consideração os parâmetros de qualidade aceitos pela indústria sementeira, como vigor de sementes e testes de germinação (Martins et al., 2006).

De maneira geral, não houve diferença estatística para a maioria das variáveis avaliadas. Também não foram observadas diferenças para interação genótipo-espaçamento para as variáveis com diferenças estatísticas.

O comprimento do ramo principal não apresentou diferença significativa, situando-se as médias nas faixas de 60,7 cm para o hibrido 02 e $46,3 \mathrm{~cm}$ para o hibrido 07 entre os materiais testados.

A diferença estatística ocorreu para as variáveis matéria fresca de parte aérea e acidez titulável (Tabela 2). Observa-se a superioridade do desenvolvimento vegetativo para os híbridos 02, 03, 06 e 07, com as médias: 521,0 g; 586,2 g; 669,1 g e 551,9 g, respectivamente. Dentre os três espaçamentos testados também não se verificaram diferenças significativas entre os mesmos. Uma das características que mais varia quando se instalam trabalhos de pesquisa envolvendo espaçamentos, segundo a literatura, é a massa média de frutos; no entanto, nesta pesquisa não se observaram alterações para a mesma entre os genótipos avaliados. Convém ressaltar que o peso dos frutos manteve a média de $2,0 \mathrm{~kg}$, visto que os genótipos experimentais, juntamente com as testemunhas, foram selecionados para plantas de pequeno porte e frutos pequenos, visando atender, principalmente, ao mercado externo. Garcia \& Sousa (2002), trabalhando com a cultivar Crimson Sweet, observaram uma diminuição linear nas massas médias de frutos (total e comercial), à 
Tabela 1. Altura da plantas $(\mathrm{cm})$ e diâmetro do colo $(\mathrm{cm})$ de genótipos diplóides experimentais de melancia (plant height $(\mathrm{cm})$ and neck diameter $(\mathrm{cm})$ of diploid watermelon experimental genotypes). Juazeiro, UNEB, 2006.

\begin{tabular}{llllll}
\hline \multirow{2}{*}{ Genótipos } & \multicolumn{2}{c}{ Altura da planta (cm) } & & \multicolumn{2}{c}{ Diâmetro do colo (cm) } \\
\cline { 2 - 3 } \cline { 5 - 6 } & $\mathbf{1 5}$ dias & $\mathbf{2 1}$ dias & & $\mathbf{1 5}$ dias & 21 dias \\
\hline Hib. 01 & $3,3 \mathrm{bcd}$ & $4,3 \mathrm{~cd}$ & & $0,18 \mathrm{~b}$ & $0,19 \mathrm{~b}$ \\
Hib. 02 & $4,6 \mathrm{a}$ & $6,1 \mathrm{a}$ & & $0,20 \mathrm{ab}$ & $0,23 \mathrm{a}$ \\
Hib. 03 & $3,9 \mathrm{abc}$ & $5,3 \mathrm{abc}$ & & $0,19 \mathrm{ab}$ & $0,21 \mathrm{ab}$ \\
Hib. 04 & $3,6 \mathrm{bcd}$ & $4,8 \mathrm{bcd}$ & & $0,19 \mathrm{ab}$ & $0,20 \mathrm{ab}$ \\
Hib. 05 & $3,6 \mathrm{bcd}$ & $4,8 \mathrm{bcd}$ & & $0,20 \mathrm{ab}$ & $0,21 \mathrm{ab}$ \\
Hib. 06 & $4,2 \mathrm{ab}$ & $5,2 \mathrm{abcd}$ & & $0,21 \mathrm{a}$ & $0,22 \mathrm{ab}$ \\
Hib. 07 & $3,8 \mathrm{abcd}$ & $4,7 \mathrm{bcd}$ & & $0,18 \mathrm{~b}$ & $0,20 \mathrm{ab}$ \\
Hib. 08 & $3,0 \mathrm{~d}$ & $4,3 \mathrm{~d}$ & & $0,20 \mathrm{ab}$ & $0,21 \mathrm{ab}$ \\
'Smile' & $4,1 \mathrm{abc}$ & $5,5 \mathrm{ab}$ & & $0,19 \mathrm{ab}$ & $0,20 \mathrm{~b}$ \\
'Sugar Baby' & $3,3 \mathrm{~cd}$ & $4,3 \mathrm{~cd}$ & & $0,17 \mathrm{~b}$ & $0,19 \mathrm{~b}$ \\
\hline CV (\%) & 19,96 & 16,57 & & 14,36 & 12,85 \\
\hline
\end{tabular}

*Médias seguidas de mesma letra na coluna não diferem significativamente pelo teste de Tukey $(\mathrm{p}<0,05)$ (means followed by same letter in the column do not differ from each other by the Tukey test, $\mathrm{p}<0.05)$.

medida que ocorreu um aumento na população de plantas. Esse comportamento não foi observado neste trabalho. Faria et al. (2000), trabalhando com melão, não observou diferença estatística entre os espaçamentos avaliados, não tendo exercido influência sobre a massa média de frutos.

Não se verificou diferenças significativas entre os genótipos testados para produtividade total. No entanto, o genótipo $06\left(40,5 \mathrm{t} \mathrm{ha}^{-1}\right)$ apresentou em magnitude rendimento superior à cv. Sugar Baby $\left(23,8 \mathrm{t} \mathrm{ha}^{-1}\right)$. Segundo NeSmith (1993), a produtividade total e comercial de frutos de melancia aumentaram quando o espaçamento entre plantas decresceu de 2,2 $\mathrm{m}$ para 0,9 m. Resende \& Costa (2003), trabalhando com a cultivar de melancia Crimson Sweet, obtiveram as maiores produções nos espaçamentos 0,6 e 0,8 $\mathrm{m}$ entre plantas na linha, 42,4 e 45,3 t ha $^{-1}$, respectivamente, não mostrando diferenças entre si.

Para número de frutos por planta e produção por planta não se observou diferença estatística situando-se, respectivamente, nas faixas de 0,5 a 1,1 e 1,7 a $3,0 \mathrm{~kg}$. Resende \& Costa (2003), trabalhando com Crimson Sweet obtiveram maior número de frutos por planta $(1,4)$ nos maiores espaçamentos $(3,0 \times 0,8 \mathrm{~m})$. Duthie et al. (1999) também observaram maior número de frutos por planta em menores densidades de plantio. Estas observações não foram corroboradas com esta pesquisa.

Tabela 2. Massa fresca da parte aérea $\left(\mathrm{g}_{\text {planta }}{ }^{-1}\right)$, produtividade $\left(\mathrm{t} \mathrm{ha}^{-1}\right)$, massa de frutos $(\mathrm{g})$ e acidez titulável $(\%)$ de genótipos de melancia diplóides experimentais plantados em diferentes espaçamentos (fresh weight of shoot $(\mathrm{g})$ yield $\left(\mathrm{t} \mathrm{ha}^{-1}\right)$, fruit mass $(\mathrm{g})$ and tritratable acidity (\%) of diploid experimental watermelon genotypes planted at different spacings). Juazeiro, UNEB, 2006.

\begin{tabular}{|c|c|c|c|c|}
\hline Tratamentos & $\begin{array}{l}\text { Massa fresca parte } \\
\text { aérea }\left(\mathrm{g} \mathrm{planta}^{-1}\right)\end{array}$ & $\begin{array}{c}\text { Produtividade comercial } \\
\qquad\left(\mathrm{t} \mathrm{ha} \mathrm{h}^{-11}\right)\end{array}$ & $\begin{array}{c}\text { Massa de frutos } \\
(\mathrm{kg})\end{array}$ & $\begin{array}{c}\mathrm{AT} \\
\text { (\% de ac. cítrico) }\end{array}$ \\
\hline \multicolumn{5}{|c|}{ Espaçamentos (m) } \\
\hline$(2,0 \times 0,3)$ & $476,1 \mathrm{a}$ & $38,3 \mathrm{a}$ & $2,2 \mathrm{a}$ & $0,11 \mathrm{a}$ \\
\hline$(2,0 \times 0,4)$ & $360,2 \mathrm{a}$ & $30,8 \mathrm{a}$ & $1,9 \mathrm{a}$ & $0,11 \mathrm{a}$ \\
\hline$(2,0 \times 0,5)$ & $500,9 \mathrm{a}$ & $21,2 \mathrm{a}$ & $2,0 \mathrm{a}$ & $0,10 \mathrm{a}$ \\
\hline \multicolumn{5}{|l|}{ Genótipos } \\
\hline Hib 01 & $434,3 b$ & $27,5 b$ & $1,8 \mathrm{a}$ & $0,08 \mathrm{c}$ \\
\hline Hib 02 & $521,0 \mathrm{a}$ & $37,9 \mathrm{e}$ & $2,4 \mathrm{a}$ & $0,09 b$ \\
\hline Hib 03 & $586,2 \mathrm{a}$ & $32,8 \mathrm{~d}$ & $2,0 \mathrm{a}$ & $0,11 b$ \\
\hline Hib 04 & $449,8 b$ & $22,7 \mathrm{a}$ & $1,9 \mathrm{a}$ & $0,10 \mathrm{~b}$ \\
\hline Hib 05 & $380,1 \mathrm{c}$ & $24,2 \mathrm{a}$ & $2,1 \mathrm{a}$ & $0,10 \mathrm{~b}$ \\
\hline Hib 06 & $669,1 \mathrm{a}$ & $40,5 \mathrm{e}$ & $2,0 \mathrm{a}$ & $0,11 \mathrm{~b}$ \\
\hline Hib 07 & $551,9 \mathrm{a}$ & $28,2 \mathrm{bc}$ & $2,2 \mathrm{a}$ & $0,11 \mathrm{~b}$ \\
\hline Hib 08 & $432,0 \mathrm{~b}$ & $32,4 d$ & $2,4 \mathrm{a}$ & $0,11 b$ \\
\hline 'Smile' & $198,1 \mathrm{c}$ & $30,8 \mathrm{~cd}$ & $1,6 \mathrm{a}$ & $0,14 \mathrm{a}$ \\
\hline 'Sugar Baby' & $256,4 \mathrm{c}$ & $23,8 \mathrm{a}$ & $1,9 \mathrm{a}$ & $0,13 \mathrm{a}$ \\
\hline $\mathrm{CV}^{1}(\%)$ & 34,18 & 18,08 & 19,07 & 0,77 \\
\hline $\mathrm{CV}^{2}(\%)$ & 18,96 & 29,28 & 17,04 & 1,12 \\
\hline
\end{tabular}

*Médias seguidas de mesma letra na coluna não diferem significativamente pelo teste de Tukey $(\mathrm{p}<0,05)$ (means followed by same letter in the column do not differ from each other by the Tukey test, $\mathrm{p}<0.05) ; \mathrm{CV}^{1}(\%)=$ parcelas; $\mathrm{CV}^{2}(\%)=$ subparcelas. 
Não houve diferença significativa para sólidos solúveis (SS) entre os diferentes espaçamentos utilizados, situando-se as médias nas faixas de 9,4 e 7,2 ${ }^{\circ}$ Brix, respectivamente, para Sugar Baby e Hib 01. Singh \& Naik (1989) não encontraram influencia significativa do espaçamento sobre sólidos solúveis em melancia, o que concorda com os resultados obtidos neste trabalho.

Para a acidez titulável houve diferença estatística entre os genótipos, sendo que as cultivares Smile e Sugar Baby revelaram os maiores valores 0,14 e 0,13 $\%$ de ácido cítrico, respectivamente. Entre os híbridos, o Hib.01 apresentou o menor valor, sendo de $0,08 \%$ de ácido cítrico (Tabela 2). A acidez devida aos ácidos orgânicos é uma característica importante no que se refere à palatabilidade de muitos frutos. Com poucas exceções, diminui com a maturação, em decorrência do processo respiratório ou de sua conversão em açúcares (Kader, 1978; Pretty, 1982).

A relação entre sólidos solúveis/ acidez titulável não sofreu alteração com os espaçamentos utilizados, nem entre os genótipos estudados, situando-se as médias nas faixas de 74,2 a 84,2 e 64,1 a 90,5, respectivamente. Essa relação é uma das melhores formas de avaliação do sabor, sendo mais representativa que a medição isolada de açúcares ou da acidez, proporcionando boa idéia do equilíbrio entre esses dois componentes (Chitarra \& Chitarra, 2005). Em algumas culturas, já foi determinada a relação que proporciona melhor sabor do fruto. Em melão, o fruto pode ser considerado adequado para o consumo quando a relação é superior a 25:1 e quando a acidez é igual ou inferior a 0,5\% (Cruess, 1973). Na melancia, Garcia (1998) obteve relações que variaram de 26,7 a 30:1, valores inferiores aos observados neste trabalho.

Entretanto, em situações em que a acidez e os sólidos solúveis são baixos, podem proporcionar relação SS/AT elevada, induzindo a interpretações errôneas com relação ao sabor do fruto.

A firmeza da polpa dos frutos não apresentou diferença significativa nos diferentes espaçamentos utilizados nem entre os genótipos avaliados, variando de $14,3 \mathrm{~N}$ na cv. Smile a $9,4 \mathrm{~N}$, no hí- brido 1. Também não houve diferença significativa para o $\mathrm{pH}$ da polpa que apresentou valores muito próximos entre os espaçamentos e genótipos, situando-se as médias na faixa de 5,2 a 5,4 e 4,8 a 5,5, respectivamente.

De maneira geral, não foram observadas diferenças com relação à qualidade dos frutos de melancia entre os híbridos e as duas cultivares Smile e Sugar Baby. Tendo em vista que os oito híbridos trabalhados estão em fase final de melhoramento e não diferiram das duas cultivares comerciais (Smile e Sugar Baby) em termos de qualidade, os mesmos se mostram bastantes promissores para o seu futuro lançamento no mercado, possibilitando aos produtores o cultivo de melancias adaptadas às condições locais.

Esses híbridos passarão por novas avaliações em épocas e locais diferentes, sendo testados a nutrição, manejo de água e qualidade pós-colheita dos genótipos. Ao final de todas essas avaliações será possível recomendar os melhores híbridos para serem comercializados e cultivados pelos produtores da região.

Quanto às características externas dos frutos e a cor da polpa, todos os genótipos testados apresentaram polpa vermelha, frutos pequenos (em torno de $2,0 \mathrm{~kg}$ ) e formato redondo. Entretanto, a casca dos frutos desses híbridos é bastante distinta da cultivar Crimson Sweet. Esta diferença é interessante pois, uma nova cultivar de frutos pequenos, para se estabelecer no mercado, não pode ser confundida com os refugos da cultivar americana que ainda domina o mercado nacional.

Os híbridos experimentais 02, 03, 04, 05 e 07 apresentaram as melhores taxas de emergência, com destaque para os híbridos 02, 03 e 06, que também estiveram entre os melhores para a altura e diâmetro do colo das plantas aos 21 dias.

Os diferentes espaçamentos utilizados não afetaram o desenvolvimento das plantas, como também não afetaram a qualidade fisiológica dos frutos dos genótipos avaliados.

\section{REFERÊNCIAS}

AGRIANUAL. 2007. São Paulo: FNP Consultoria \& Comércio. p.544.
ANDRADE JUNIOR AS. 2004. A cultura da melancia. Brasília, DF: Embrapa-SPI/ Teresina: Embrapa-CPAMN. 86p. (Coleção Plantar, 34).

BRASIL. Ministério da Agricultura e Reforma Agrária. 1992. Regras para análise de sementes. Brasília: SNDA/DNDV/CLAV, $365 \mathrm{p}$.

BRINEN GE; LOCASCIO SJ; ELMSTROM GW. 1979. Plant arrangement for increased watermelon yield. Selected Proceedings of the Florida State Horticultural Society 92: 80-82.

BRINEN GH; LOCASCIO SJ. 1979. Plant and row spacing, mulch, and fertilizer rate effects on watermelon production. Journal of the American Society for Horticultural Science 104: 724-726.

CHITARRA MI; CHITARRA AB. 2005. Póscolheita de frutos e hortaliças: fisiologia e manuseio. 2. ed. ver. e ampl. Lavras: UFLA, $785 \mathrm{p}$.

COMISSÃO ESTADUAL DE FERTILIDADE DO SOLO. 1989. Manual de adubação e calagem para o Estado da Bahia. Salvador: CEPLAC/EMATERBA/EMBRAPA/EPABA /NITROFERTIL, $176 \mathrm{p}$.

CRUESS WV. 1973. Produtos industriais de frutos e hortaliças. São Paulo: Edgard Blücher, v. $1,446 \mathrm{p}$.

DIAS RCS; COSTAND; QUEIRÓZ MA; FARIA CMB. 2001. Cultura da Melancia. Petrolina: Embrapa Semi-Árido. 20p. (Circular Técnica, 63)

DUTHIE JA; ROBERTS BW; EDELSON JV; SHREFLER JW 1999. Plant densitydependent variation in density, frequency, and size of watermelon fruits. Crop Science. 39: 406-412.

FARIA CM; COSTA ND; PINTO JM. 2000. Níveis de nitrogênio por fertirrigação e densidade de plantio na cultura do melão em um Vertissolo. Pesquisa Agropecuária Brasileira 35: 491495.

FERREIRA MAJF; QUEIROZ MA; BRAZ LT. 2003. Correlações genotípicas, fenotípicas e de ambiente entre dez caracteres de melancia e suas implicações para o melhoramento genético. Horticultura Brasileira 21: 438-442.

GARCIA LF. 1998. Influência do espaçamento e da adubação nitrogenada sobre a produtividade da melancia no baixo Parnaíba Piauiense. Comunicado Técnico 79, Embrapa Meio Norte, $5 \mathrm{p}$.

GARCIA LF; SOUZA VAB. 2002. Influência do espaçamento e da adubação nitrogenada sobre a produção da melancia. Revista de la Facultad de Agronomia 28: 59-70.

HALSEY LH. 1959. Watermelon spacing and fertilization. Proceedings State Horticultural Society 72: 131-135.

HEDGE DM. 1988. Effect of irrigation and nitrogen fertilization on yield, quality, $\mathrm{N}$ uptake and water use of watermelon (Citrullus lanatus). Indian Journal of Agricultural Science 58: 444-448.

KADER AA. 1978. Quality factors: definition and evaluation for fresh horticultural crops. In: KADER AA. Postharvest Technology of Horticultural Crops. California: University 
of California. p.118-121.

MARTINS CC; CASTRO MM; SENEME AM; NAKAGAWA J. 2006. Metodologia para avaliação do vigor de sementes de tomate. Horticultura Brasileira 24: 301-304.

NESMITH DS. 1993. Plant spacing influences watermelon yield and yield components. HortScience 28: 885-887.

PATIL CB; BHOSALE RJ. 1976. Effect of nitrogen fertilization and spacing on the yield of watermelon. Indian Journal of Agronomy 21: 300-301.

PRETTY KM. 1982. O potássio e a qualidade da produção agrícola. In: YAMADA T; IGUE K; MUZILLI O; USHERWOOD NR (eds) Potássio na agricultura brasileira. Piracicaba: POTAFOS. p.177-194.
RESENDE GM; COSTAND. 2003. Características produtivas da melancia em diferentes espaçamentos de plantio. Horticultura Brasileira 21: 695-698.

SANTOS FILHAMEC; MORAIS FA;ANDRADE MEL; ARAÚJO JMM; AROUCHA EMM. 2005. Caracterização pós-colheita de híbridos de melancia sem semente nas condições de Mossoró-RN. In: CONGRESSO BRASILEIRO DE OLERICULTURA, 45. Anais eletrônicos. Disponível em: http:// www.abhorticultura.com.br. Acessado em agosto de 2007.

SANTOS JA; FACTOR TL; VILLELA JUNIOR LVE; ARAÚJO JAC. 2002. Produção de melancia tutorada sob diferentes densidades de plantio em ambiente protegido. In: CONGRESSO BRASILEIRO DE OLERICULTURA, 42. e CONGRESSO LATINO AMERICANO DE HORTICULTURA, 11. Resumos. Horticultura Brasileira 20:315, resumo 261, Suplemento 1.

SINGH RV; NAIK LB. 1989. Response of watermelon (Citrullus lanatus Thumbs. Monsf.) to plant density, nitrogen and phosphorus fertilization. Indian Journal of Horticulture 46: 80-83.

SRINIVAS K; HEDGE DM; HAVANAGI GV. 1991. Effect of nitrogen fertilization and plant population on plant water relations, canopy temperature, yield and water use efficiency of watermelon (Citrullus lanatus). Singapore. Journal of Primary Industries 19: 8-15. 